\title{
COMPORTAMIENTO FISIOLÓGICO DE LA PERA VARIEDAD TRIUNFO DE VIENA (Pyrus Communis L.) DURANTE EL PERÍODO POSCOSECHA ${ }^{1}$
}

\author{
ALFONSO PARRA-CORONADO², JOSÉ EUGENIO HERNÁNDEZ HERNÁNDEZ \\ JESÚS HERNÁN CAMACHO-TAMAYO ${ }^{4}$
}

\begin{abstract}
RESUMEN - La pera variedad Triunfo de Viena es una fruta producida en Colombia y actualmente no se dispone de una red de frío para su manejo poscosecha. El objetivo de este trabajo fue establecer el comportamiento fisiológico de la pera variedad Triunfo de Viena en tres condiciones de temperatura y humedad relativa: $3^{\circ} \mathrm{C}$ y $85 \%, 11^{\circ} \mathrm{C}$ y $80 \%, 18^{\circ} \mathrm{C}$ y $75 \%$. La pera variedad Triunfo de Viena es un fruto climatérico, cuyo punto de máxima intensidad respiratoria puede desplazarse en el tiempo dependiendo de las condiciones de almacenamiento. Para las condiciones estudiadas, el climaterio se presentó en el día $22\left(35,87 \mathrm{mg} \mathrm{CO}_{2} \mathrm{~kg}^{-1} \mathrm{~h}^{-1}\right)$, en el día $36\left(28,59 \mathrm{mg} \mathrm{CO}_{2} \mathrm{~kg}^{-1} \mathrm{~h}^{-1}\right)$ y en el día $93\left(15,72 \mathrm{mg} \mathrm{CO}_{2} \mathrm{~kg}^{-1} \mathrm{~h}^{-1}\right)$ del almacenamiento, para las temperaturas de $18^{\circ} \mathrm{C}, 11^{\circ} \mathrm{C}$ y $3^{\circ} \mathrm{C}$ respectivamente. La pérdida de peso presentó una relación lineal y directa respecto al tiempo de almacenamiento, siendo mayor con temperaturas de $18^{\circ} \mathrm{C} \mathrm{y} 11^{\circ} \mathrm{C}$. La acidez titulable y el $\mathrm{pH}$ no presentan grandes variaciones durante el almacenamiento. La intensidad respiratoria, la pérdida de peso, la relación SST/AT y la firmeza de la pulpa, son las características que representan mejor la evolución de la madurez de las peras variedad Triunfo de Viena.
\end{abstract}

Términos para indexación: Propiedades físicas, almacenamiento, índices de madurez, calidad.

\section{PHYSIOLOGICAL PERFORMANCE OF PEARS (Pyrus Communis L.), cv. TRIUNFO DE VIENA DURING POSTHARVEST PERIOD}

\begin{abstract}
The pear cv. Triunfo de Viena is a fruit produced in Colombia, but at present time there is no cool chamber to handle it. The objective of this work was to determine the pear's physiological performance under three conditions of temperature and relative humidity: $3^{\circ} \mathrm{C}$ and $85 \%, 11^{\circ} \mathrm{C}$ and $80 \%, 18^{\circ} \mathrm{C}$ and $75 \%$. The pear cv. Triunfo de Viena is a climacterically fruit, which its maximum point of respiratory rate could vary according to the storage conditions. For the evaluated conditions, the number of days required to reach the climacteric-like peak was $22\left(35,87 \mathrm{mg} \mathrm{CO}_{2} \mathrm{~kg}^{-1} \mathrm{~h}^{-1}\right)$, $36\left(28,59 \mathrm{mg} \mathrm{CO}_{2} \mathrm{~kg}^{-1} \mathrm{~h}^{-1}\right)$ and $93\left(15,72 \mathrm{mg} \mathrm{CO}_{2} \mathrm{~kg}^{-1} \mathrm{~h}^{-1}\right)$, at temperatures of $18^{\circ} \mathrm{C}, 11^{\circ} \mathrm{C}_{\text {and }} 3^{\circ} \mathrm{C}$ respectively. The weight loss presented a linear and direct relation in regard to the storage period, being greater with temperatures of $18^{\circ} \mathrm{C}$ and $11^{\circ} \mathrm{C}$. The titratable acids and $\mathrm{pH}$ do not present great variations during the storage. The respiratory rate, the weight loss, the SST/AT relation and the flesh firmness are characteristics that better represent the evolution of the ripening pears cv. Triunfo de Viena.
\end{abstract}

Index terms: physical properties, storage, maturity index, quality.

\section{INTRODUCCIÓN}

En Colombia como en la mayoría de los países en vía de desarrollo, existen dificultades para satisfacer las necesidades alimenticias de la población y en particular para que esta pueda tener acceso a productos de buena calidad, a precios razonables. Debido a la falta de capacitación y de organización de los agricultores de economía campesina y al desconocimiento de prácticas tecnológicas apropiadas antes, durante y después de la cosecha, en Colombia se pierde entre el 40 y el $60 \%$ de la producción de frutas, correspondiendo aproximadamente a 1,25 millones de toneladas (Parra, 1997).

La mayor parte de las operaciones de acondicionamiento poscosecha tales como limpieza, selección y clasificación aún no se realizan en forma técnica y eficiente a pesar de que el Instituto Colombiano de Normas Técnicas (ICONTEC), cuenta con un paquete de recomendaciones al respecto que cubre no menos de 50 productos frutícolas (Norma GTC 66, 1999).

El proceso de respiración de las frutas es una combinación entre la producción de dióxido de carbono y consumo de oxígeno, mediante procesos de difusión celular (Schouten et al., 2004). La inhibición del proceso respiratorio incide directamente en el sostenimiento de la calidad de frutas y hortalizas y puede realizarse por medio del manejo de atmósferas controladas o modificadas (Amarante \& Banks, 2001), así como mediante el control de la temperatura y la humedad relativa de almacenamiento (Coutinho et al., 2003).

La pera es un producto climatérico y debe cosecharse cuando la intensidad respiratoria presenta un valor mínimo, con el fin de facilitar su manejo poscosecha y prolongar el tiempo de almacenamiento, retardando el climaterio y disminuyendo los cambios fisiológicos como la hidrólisis del almidón, aumento de los sólidos solubles totales, pérdida de color y la transformación de la propectina, responsable por la turgencia y pérdida de firmeza de la pulpa (Pantastico, 1979).

De acuerdo con Barragán \& Sánchez (1998), en Colombia el manejo poscosecha de la pera variedad Triunfo de Viena no es eficiente, ya que no se dispone de una red de frío por parte de los agricultores y comercializadores. La cosecha se realiza entre los meses de marzo y junio, donde los precios de venta son bajos, incidiendo en una reducción de la rentabilidad para los productores. Por esto, el objetivo del presente trabajo fue evaluar la calidad de la pera variedad Triunfo de Viena, bajo tres condiciones de almacenamiento, con el fin de aumentar el tiempo de comercialización de esta variedad.

\section{MATERIALYMÉTODOS}

La materia prima para la realización de este estudio fue la pera variedad Triunfo de Viena, cuyas muestras se tomaron de 10 fincas diferentes del municipio de Nuevo Colón, ubicado en el Departamento de Boyacá (zona Andina Colombiana), en las coordenadas geográficas $5^{\circ} 21^{\prime} 11^{\prime}$ "de latitud norte, $73^{\circ} 27^{\prime} 24^{\prime}$ ' de longitud oeste y una altitud de $2464 \mathrm{~m}$. El transporte de la materia prima desde la finca al lugar de almacenamiento (Laboratorio de Frutas y Hortalizas del Departamento de Ingeniería Agrícola de la Universidad Nacional de Colombia, sede Bogotá), se efectuó en recipientes plásticos de 0,60 m de largo, 0,30 m de ancho y $0,25 \mathrm{~m}$ de alto y una capacidad de $20 \mathrm{~kg}$. Se realizó una

\footnotetext{
${ }^{1}$ (Trabalho 124/2005). Recebido: 16/08/2005. Aceito para publicação: 30/03/2006.

2 Ing. Agrícola, M. Sc., Universidad Nacional de Colombia, Profesor Titular, programa de Ingeniería Agrícola, Ciudad Universitaria, Bogotá, Colombia. aparrac@unal.edu.co.

${ }^{3}$ Ing. Agrícola, M. Sc., Universidad Nacional de Colombia, Profesor Asociado, programa de Ingeniería Agrícola, Ciudad Universitaria, Bogotá, Colombia. jehernandezh@unal.edu.co.

${ }^{4}$ Ing. Agrícola, M. Sc., Universidad Nacional de Colombia, Profesor Asistente, programa de Ingeniería Agrícola, Ciudad Universitaria, Bogotá, Colombia. jhcamachot@unal.edu.co.
} 
selección previa con el fin de garantizar la sanidad del producto.

Se tomaron 500 unidades el día de la cosecha, de las cuales se seleccionaron 375 que se distribuyeron de la siguiente manera: 75 individuos se almacenaron a una temperatura de $18{ }^{\circ} \mathrm{C}$ y humedad relativa de $75 \%$ (T3); 100 individuos se almacenaron a una temperatura de $11{ }^{\circ} \mathrm{C}$ y humedad relativa de $80 \%$ (T2) y 146 individuos se almacenaron a una temperatura de $3{ }^{\circ} \mathrm{C}$ y humedad relativa de $85 \%$ (T1).

El peso fue determinado mediante una balanza electrónica marca Mettler PC2000, precisión 0,01g. Los índices de madurez determinados durante el estudio fueron: firmeza de la pulpa $(\mathrm{N})$, mediante un penetrómetro de reloj marca BERTUZZI FT 327 con escala de 0 a 130 $\mathrm{N}$ y precisión de $1,0 \mathrm{~N}$, con punta de 11,25 mm de diámetro; acidez titulable (AT), expresada como \% de ácido málico; sólidos solubles totales - SST ( ${ }^{\circ}$ Brix), usando un refractómetro manual marca KIKUCHI, con escala 0 a $30^{\circ}$ Brix; relación de madurez $(\mathrm{RM})$, determinada mediante la relación SST/AT; $\mathrm{pH}$, utilizando un potenciómetro digital, el cual indica directamente la lectura del $\mathrm{pH}$; intensidad respiratoria - IR (mg $\left.\mathrm{CO}_{2} \mathrm{~kg}^{-1} \mathrm{~h}^{-1}\right)$, para lo cual se utilizaron los respirómetros, en los cuales se colocaron $30 \mathrm{ml}$ de hidróxido de bario $\left(\mathrm{Ba}(\mathrm{OH})_{2} 2 \mathrm{H}_{2} \mathrm{O}\right)$ con normalidad de 0,1 como reactivo de captura del $\mathrm{CO}_{2}$ generado en la respiración, dejándose funcionar por 30 minutos, al cabo de los cuales se extrajo una muestra de $10 \mathrm{ml}$ del hidróxido de bario, el cual se tituló con ácido oxálico $\left(\mathrm{H}_{2} \mathrm{C}_{2} \mathrm{O}_{4}-2 \mathrm{H}_{2} \mathrm{O}\right)$ con normalidad 0,1 , para una muestra aproximada de $1,0 \mathrm{~kg}$ de pera colocados en la cámara de respiración. La intensidad respiratoria se determinó mediante la siguiente ecuación:

$$
\boldsymbol{I R}=\frac{(\boldsymbol{V b}-\boldsymbol{V m}) * N^{*} 22 \boldsymbol{m g} \mathrm{CO}_{2} \boldsymbol{m e q}}{\boldsymbol{W} * \boldsymbol{t}}
$$

Donde,

IR: Intensidad respiratoria en $\mathrm{mg}$ de $\mathrm{CO}_{2} \mathrm{~kg}^{-1} \mathrm{~h}^{-1}$

Vb: Volumen de ácido oxálico, gastados al titular el blanco $\left(\sin \mathrm{CO}_{2}\right), \mathrm{ml}$. Vm: Volumen de ácido oxálico, gastados al titular la muestra, $\mathrm{ml}$.

$\mathrm{N}$ : Normalidad del ácido oxálico.

$\mathrm{W}$ : Peso de la muestra del fruto, $\mathrm{kg}$.

t: Tiempo, h.

El diseño estadístico fue enteramente casualizado, utilizando 25 frutas para la pérdida de peso, 3 frutas y 3 repeticiones para la determinación de la firmeza de la pulpa, pH, SST y AT. Para la IR se realizaron cinco repeticiones, con muestras cercanas a $0,5 \mathrm{~kg}$. Para los resultados encontrados, se realizaron análisis de varianza y comparación de medias, mediante prueba de Duncan, a un nivel del 5\% de probabilidad.

\section{RESULTADOS Y DISCUSIÓN}

El peso de la pera variedad Triunfo de Viena disminuye con el

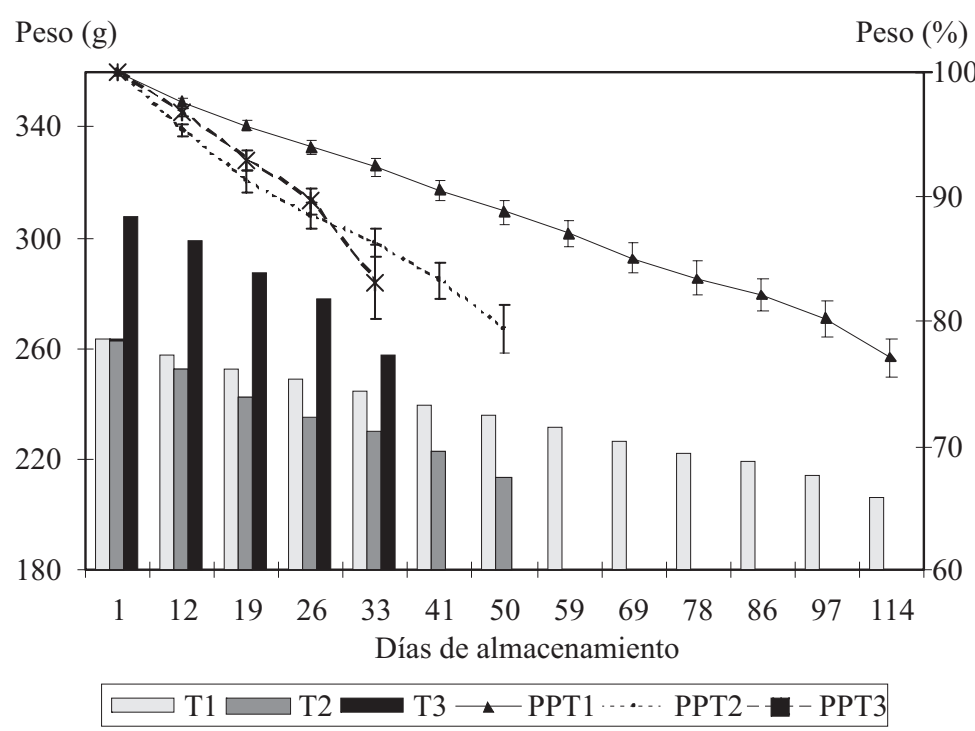

FIGURA 1 - Variación media del peso en los tres ambientes de almacenamiento. $\mathrm{T} 1=$ fruto almacenado a $3^{\circ} \mathrm{C}$ y humedad relativa del $85 \% ; \mathrm{T} 2=$ fruto almacenado a $11^{\circ} \mathrm{C}$ y humedad relativa del $80 \%$; T3 $=$ fruto almacenado a $18^{\circ} \mathrm{C}$ y humedad relativa del $75 \%$. $\mathrm{PP}=$ peso del fruto en porcentaje. Las barras representan el intervalo de confianza del $95 \%$ para la media.

tiempo, mostrando una relación lineal directa con el tiempo de almacenamiento, como también lo reporta Lombardi et al. (2000) para la variedad Shinsseiki. En el periodo poscosecha se observa que las condiciones más adversas para su conservación corresponden a la mayor temperatura de almacenamiento, $18^{\circ} \mathrm{C}$ y menor $\mathrm{HR}$ de $75 \%$ (Figura 1). Para esta condición, el deterioro se presentó a partir del día 20 de almacenamiento, manifestándose por el inicio de pudrición en la base, manchas y ataque de hongos, con una pérdida final de peso del $16,9 \%$ durante 33 días de almacenamiento. Los productos almacenados a $11^{\circ} \mathrm{C}$ y $80 \%$ de humedad relativa, presentaron una pérdida final de peso del $20,16 \%$ durante 50 días de almacenamiento. Los productos almacenados a $3^{\circ} \mathrm{C}$ y $85 \%$ de humedad relativa, iniciaron su deterioro en el día 85 del almacenamiento, llegando a ser total a los 114 días con una pérdida final de peso de $22,61 \%$. Se observa que a menor HR, la pérdida es mayor, situación normal en frutas, ya que la pérdida de agua de estos productos es mayor cuando la HR del ambiente en que se encuentran es baja.

La pérdida de peso va acompañada también de disminución de la pérdida de firmeza de la pulpa. Para las condiciones de almacenamiento consideradas, la firmeza de la pera disminuye a través del tiempo, siendo más rápida en los productos almacenados a temperaturas más elevadas (Tabla 1); observándose que la firmeza es

TABLA 1 - Valores medios de la firmeza de la pulpa de la pera variedad Triunfo de Viena, bajo tres condiciones de almacenamiento.

\begin{tabular}{|c|c|c|c|c|c|c|c|c|c|}
\hline \multirow{2}{*}{ DÍA } & \multicolumn{3}{|c|}{ T1 } & \multicolumn{3}{|c|}{ T2 } & \multicolumn{3}{|c|}{ T3 } \\
\hline & Med & & CV, \% & Mec & & CV, \% & Me & & $\mathrm{CV}, \%$ \\
\hline 1 & 70,32 & 1 & 8,25 & 70,32 & 1 & 8,25 & 70,32 & 1 & 8,25 \\
\hline 12 & 65,78 & 1 & 9,38 & 66,60 & 1 & 8,76 & 28,61 & $\mathrm{~g}$ & 7,47 \\
\hline 19 & 65,89 & 1 & 8,61 & 19,02 & ef & 28,59 & 13,84 & cde & 19,95 \\
\hline 26 & 67,91 & 1 & 11,70 & 16,35 & de & 30,74 & 10,46 & $\mathrm{~cd}$ & 44,47 \\
\hline 33 & 66,27 & 1 & 8,00 & 11,12 & $\mathrm{~cd}$ & 12,48 & 7,85 & $\mathrm{bc}$ & 41,46 \\
\hline 41 & 71,07 & 1 & 18,73 & 2,56 & $a b$ & 72,78 & & & \\
\hline 50 & 69,44 & 1 & 5,96 & 0,38 & $\mathrm{a}$ & 154,52 & & & \\
\hline 59 & 64,36 & 1 & 12,16 & & & & & & \\
\hline 69 & 56,14 & $\mathrm{k}$ & 20,71 & & & & & & \\
\hline 78 & 47,69 & $\mathrm{j}$ & 30,06 & & & & & & \\
\hline 86 & 38,37 & hi & 10,02 & & & & & & \\
\hline 97 & 44,31 & ij & 20,33 & & & & & & \\
\hline 100 & 37,22 & $\mathrm{~h}$ & 18,12 & & & & & & \\
\hline 114 & 24,85 & fg & 23,35 & & & & & & \\
\hline
\end{tabular}

Medias seguidas de letras distintas indican diferencias, según prueba de Duncan $(P<0,05)$. T1 $=$ fruto almacenado a $3^{\circ} \mathrm{C}$ y humedad relativa del $85 \%$; $22=$ fruto almacenado a $11^{\circ} \mathrm{C}$ y humedad relativa del $80 \%$; $\mathrm{T} 3=$ fruto almacenado a $18^{\circ} \mathrm{C}$ y humedad relativa de $75 \%$. 
mayor conforme menor sea la temperatura de almacenamiento. Resultados similares fueron encontrados por Trinchero et al. (2004), Coutinho et al. (2003) y por Amarente et al. (2001) con peras variedad Bartlett, Carrick y Comice respectivamente, sometidas a diferentes condiciones de almacenamiento. Según Ahmede y Labavith (1970), citados por Seibert et al (2000), la disminución de la firmeza de la pulpa es debida básicamente a la disociación de las paredes celulares, con disminución en el grado de polimerización de ácidos urónicos, que generalmente están acompañados por aumento de los contenidos de pectinas solubles.

En términos generales a menor temperatura y mayor HR, la firmeza de la pulpa de la pera no varia significativamente por periodos de hasta 90 días, pero una vez que se cambian estas condiciones y el producto se expone a temperaturas cercanas a $20^{\circ} \mathrm{C}$ y con humedad relativa inferior al $75 \%$, la firmeza se reduce drásticamente, reduciendo la calidad de la pera por periodos que pueden variar entre 5 y 11 días (Calvo, 2004; Trinchero et al., 2004; Ma y Chen, 2003; Amarante et al., 2001). Mwanaki et al. (2005) observaron una caída rápida de la firmeza de la pulpa, a partir del momento en que se presentó el climaterio de la pera Variedad "Le France".

La intensidad respiratoria para la pera (variedad Triunfo de Viena) en las diferentes condiciones de almacenamiento (Figura 2), presentó el comportamiento típico de productos climatéricos, lo cual está de acuerdo con lo reportado por diferentes autores (Mwaniki et al., 2005; Trinchero et al., 2004; Ma y Chen, 2003; Planella, 1987). En los días posteriores a la cosecha presentó un aumento paulatino de la intensidad respiratoria hasta llegar al climaterio, a partir del cual la intensidad respiratoria comienza a descender indicando el inicio de la senescencia del fruto. La magnitud de estos cambios en la tasa de respiración estuvo en función de las condiciones de almacenamiento, como se observa en la Figura 2. Las frutas almacenadas a una temperatura de $18^{\circ} \mathrm{C}$ y Humedad Relativa de $75 \%$, llegaron al climaterio a los 22 días de almacenadas con una intensidad respiratoria de $35,87 \mathrm{mg} \mathrm{CO}_{2} \mathrm{~kg}^{-1} \mathrm{~h}$ ${ }^{1}$. La intensidad respiratoria descendió hasta $15,21 \mathrm{mg} \mathrm{CO}_{2} \mathrm{~kg}^{-1} \mathrm{~h}^{-1}$; en el día 33 se observó un deterioro total del producto. Los frutos de pera almacenados a temperatura de $11^{\circ} \mathrm{C}$ y humedad relativa de $80 \%$, presentaron el climaterio a los 36 días de almacenamiento con una intensidad respiratoria de $28,59 \mathrm{mg} \mathrm{CO}_{2} \mathrm{~kg}^{-1} \mathrm{~h}^{-1}$. Para los productos almacenados a una temperatura de $3^{\circ} \mathrm{C}$ y humedad relativa de $85 \%$, el climaterio no se observa de manera clara, presentando un pico a los 93 días de almacenamiento con una intensidad respiratoria de $15,72 \mathrm{mg}$ $\mathrm{CO}_{2} \mathrm{~kg}^{-1} \mathrm{~h}^{-1}$. De acuerdo a los valores de la intensidad respiratoria registrados para $3^{\circ} \mathrm{C}$, se observa que la tasa de respiración disminuye significativamente para el periodo de almacenamiento y de ahí el aumento de la vida del producto. Ma y Chen (2003) para la variedad Comice, reportan que a $-1{ }^{\circ} \mathrm{C}$, la IR disminuye drásticamente, presentando dificultad para su medición, observando que al pasar los productos a un ambiente de $20^{\circ} \mathrm{C}$, entre 9 y 12 días después presentaron un climaterio definido.

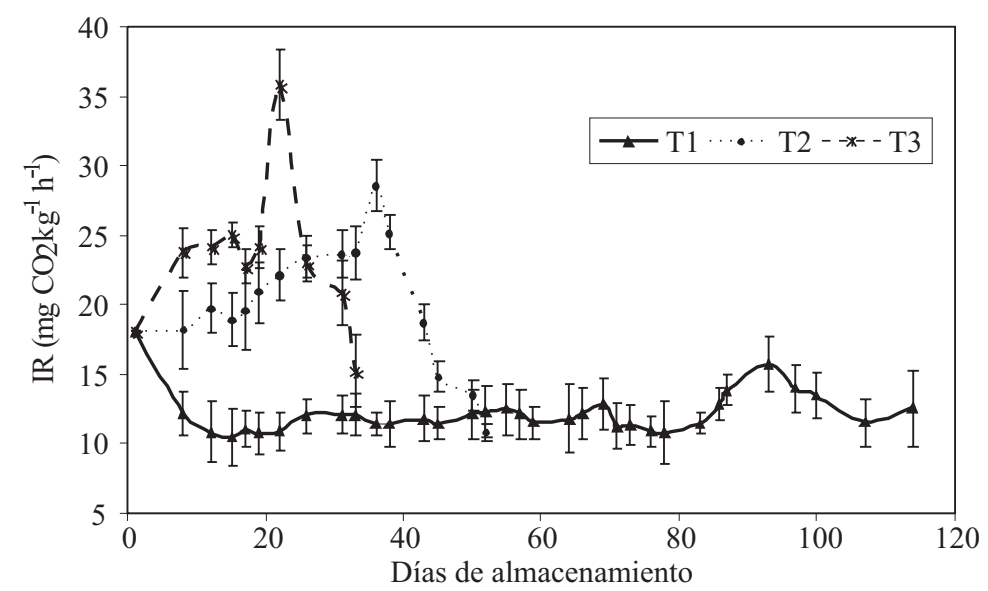

FIGURA 2 - Variación media de la IR de la pera variedad Triunfo de Viena en las tres condiciones de almacenamiento. T1= fruto almacenado a $3^{\circ} \mathrm{C}$ y humedad relativa del $85 \%$; $\mathrm{T} 2=$ fruto almacenado a $11^{\circ} \mathrm{C}$ y humedad relativa del $80 \%$; $\mathrm{T} 3=$ fruto almacenado a $18^{\circ} \mathrm{C}$. Las barras representan el intervalo de confianza del $95 \%$ para la media.

Por otra parte, algunos frutos almacenados a $3^{\circ} \mathrm{C}$ empezaron a sufrir daño por frío a los 85 días, manifestándose por el oscurecimiento interno de la pulpa, el cuál se hizo más crítico después del climaterio. El almacenamiento se prolongó hasta 114 días, con una intensidad respiratoria de $12,55 \mathrm{mg} \mathrm{CO} \mathrm{kg}^{-1} \mathrm{~h}^{-1}$, donde se observó que aproximadamente un $60 \%$ de las peras presentaban deshidratación y oscurecimiento interno debido a la exposición prolongada a bajas temperatura. Esta situación es reportada también para la pera asiática "Ya Li" (Brackmann et al., 2002), cuando es almacenada a bajas temperaturas durante 52 días.

Los valores medios de $\mathrm{pH}$ oscilan entre 4,04 y 4,90 aproximadamente para todas las condiciones de almacenamiento (Tabla 2), observándose un incremento a través del tiempo, pero sin mostrar diferencias significativas entre los tratamientos, para el mismo día, en la mayoría de los casos. Esta situación concuerda con el comportamiento de los frutos en general, los cuales al ir madurando presentan disminución del contenido de ácidos y aumento del contenido de sólidos solubles totales. Por otra parte, los mayores valores de $\mathrm{pH}$ se presentaron en la senescencia de los productos, es decir, después de alcanzar el climaterio.

Para las diferentes condiciones de almacenamiento, se observa que la acidez titulable disminuye a medida que el fruto madura (Tabla 3), sin presentar diferencias significativas marcadas para un mismo día.

TABLA 2 - Valores medios del pH de la pera variedad Triunfo de Viena, bajo tres condiciones de almacenamiento.

\begin{tabular}{|c|c|c|c|c|c|c|c|c|c|}
\hline \multirow{3}{*}{$\begin{array}{c}\text { DíA } \\
1\end{array}$} & \multicolumn{3}{|c|}{ T1 } & \multicolumn{3}{|c|}{ T2 } & \multicolumn{3}{|c|}{ T3 } \\
\hline & \multicolumn{2}{|c|}{ Media } & \multirow{2}{*}{$\frac{\mathbf{C V}, \boldsymbol{\%}}{3,37}$} & \multicolumn{2}{|c|}{ Media } & \multirow{2}{*}{$\begin{array}{c}\mathbf{C V}, \boldsymbol{\%} \\
3,37\end{array}$} & \multicolumn{2}{|c|}{ Media } & \multirow{2}{*}{$\begin{array}{r}\mathbf{C V}, \% \\
3,37\end{array}$} \\
\hline & 4,16 & abcd & & 4,16 & abcd & & 4,16 & abcd & \\
\hline 12 & 4,04 & $\mathrm{a}$ & 2,48 & 4,08 & $a b$ & 4,14 & 4,21 & abcd & 2,90 \\
\hline 19 & 4,07 & $a b$ & 2,12 & 4,07 & $a b$ & 1,58 & 4,26 & abcd & 2,24 \\
\hline 26 & 4,25 & abcd & 3,06 & 4,36 & abcde & 3,67 & 4,32 & abcde & 0,67 \\
\hline 33 & 4,09 & $a b c$ & 5,51 & 4,39 & bcde & 1,60 & 4,37 & bcde & 0,95 \\
\hline 41 & 4,46 & def & 5,20 & 4,90 & g & 1,54 & & & \\
\hline 50 & 4,41 & cde & 6,61 & 4,70 & fg & 4,34 & & & \\
\hline 59 & 4,15 & abcd & 6,93 & & & & & & \\
\hline 69 & 4,25 & abcd & 2,81 & & & & & & \\
\hline 78 & 4,29 & abcd & 4,19 & & & & & & \\
\hline 86 & 4,61 & ef & 2,32 & & & & & & \\
\hline 97 & 4,32 & bcde & 2,36 & & & & & & \\
\hline 114 & 4,74 & fg & 6,06 & & & & & & \\
\hline
\end{tabular}

Medias seguidas de letras distintas indican diferencias, según prueba de Duncan $(P<0,05)$. T1 $=$ fruto almacenado a $3^{\circ} \mathrm{C}$ y humedad relativa del $85 \%$; $22=$ fruto almacenado a $11^{\circ} \mathrm{C}$ y humedad relativa del $80 \%$; T3= fruto almacenado a $18^{\circ} \mathrm{C}$. 
TABLA 3 - Valores medios de la AT de la pera variedad Triunfo de Viena, bajo tres condiciones de almacenamiento.

\begin{tabular}{|c|c|c|c|c|c|c|c|c|c|}
\hline \multirow{2}{*}{ DÍA } & \multicolumn{3}{|c|}{ T1 } & \multicolumn{3}{|c|}{ T2 } & \multicolumn{3}{|c|}{ T3 } \\
\hline & \multicolumn{2}{|c|}{ Media (\%AM) } & \multirow{2}{*}{$\frac{\mathbf{C V}, \%}{6,45}$} & \multicolumn{2}{|c|}{ Media (\%AM) } & \multirow{2}{*}{$\frac{\mathbf{C V}, \boldsymbol{\%}}{6,45}$} & \multicolumn{2}{|c|}{ Media (\%AM) } & $\mathrm{CV}, \%$ \\
\hline 1 & 0,31 & $\mathrm{~g}$ & & 0,31 & $\mathrm{~g}$ & & 0,31 & $\mathrm{~g}$ & 6,45 \\
\hline 12 & 0,29 & efg & 3,45 & 0,25 & cdef & 12,06 & 0,27 & defg & 12.85 \\
\hline 19 & 0,26 & cdef & 4,50 & 0,25 & cdef & 9,94 & 0,17 & $\mathrm{a}$ & 3.33 \\
\hline 26 & 0,31 & $\mathrm{~g}$ & 3,23 & 0,24 & bcde & 4,17 & 0,23 & bcd & 15.68 \\
\hline 33 & 0,29 & efg & 9,12 & 0,23 & bcd & 17,32 & 0,20 & $a b$ & 11.74 \\
\hline 41 & 0,31 & $\mathrm{~g}$ & 13,29 & 0,21 & $a b c$ & 16,99 & & & \\
\hline 50 & 0,25 & cdef & 2,28 & 0,19 & $\mathrm{ab}$ & 9,12 & & & \\
\hline 59 & 0,30 & $\mathrm{fg}$ & 10,84 & & & & & & \\
\hline 69 & 0,23 & bcd & 16,70 & & & & & & \\
\hline 78 & 0,23 & bcd & 6,74 & & & & & & \\
\hline 86 & 0,22 & $a b c$ & 15,75 & & & & & & \\
\hline 97 & 0,22 & $a b c$ & 14,10 & & & & & & \\
\hline 114 & 0,21 & $a b c$ & 15,55 & & & & & & \\
\hline
\end{tabular}

Medias seguidas de letras distintas indican diferencias, según prueba de Duncan $(P<0,05)$. T1 $=$ fruto almacenado a $3^{\circ} \mathrm{C}$ y humedad relativa del $85 \%$; $22=$ fruto almacenado a $11^{\circ} \mathrm{C}$ y humedad relativa del $80 \%$; T3= fruto almacenado a $18^{\circ} \mathrm{C}$.

TABLA 4 - Valores medios de los SST de la pera variedad Triunfo de Viena, bajo tres condiciones de almacenamiento.

\begin{tabular}{|c|c|c|c|c|c|c|c|c|c|}
\hline \multirow{2}{*}{ DÍA } & \multicolumn{3}{|c|}{$\begin{array}{r}\mathrm{T} 1 \\
\end{array}$} & \multicolumn{3}{|c|}{ T2 } & \multicolumn{3}{|c|}{ T3 } \\
\hline & \multicolumn{2}{|c|}{ Media $\left({ }^{\circ}\right.$ Brix $)$} & \multirow{2}{*}{$\begin{array}{c}\mathbf{C V}, \mathbf{\%} \\
11,29\end{array}$} & \multicolumn{2}{|c|}{ Media ( ${ }^{\circ}$ Brix) } & \multirow{2}{*}{$\begin{array}{c}\mathbf{C V}, \mathbf{\%} \\
11,29\end{array}$} & \multicolumn{2}{|c|}{ Media ( ${ }^{\circ}$ Brix) } & \multirow{2}{*}{$\frac{\mathrm{CV}, \boldsymbol{\%}}{11,29}$} \\
\hline 1 & 12,67 & $a b c$ & & 12,67 & $a b c$ & & 12,67 & $a b c$ & \\
\hline 12 & 12,00 & $a b c$ & 2,89 & 14,10 & cdefg & 3,25 & 14,53 & $\mathrm{fg}$ & 7,06 \\
\hline 19 & 12,13 & $a b$ & 0,95 & 13,27 & abcdefg & 2,30 & 12,33 & $\mathrm{ab}$ & 4,68 \\
\hline 26 & 12,73 & abcd & 10,69 & 14,27 & cdefg & 4,51 & 14,47 & efg & 13,85 \\
\hline 33 & 12,80 & abcde & 4,13 & 13,80 & bcdefg & 3,83 & 12,80 & abcde & 3,13 \\
\hline 41 & 12,87 & abcdef & 12,56 & 12,33 & $\mathrm{ab}$ & 2,48 & & & \\
\hline 50 & 13,27 & abcdefg & 6,09 & 13,00 & abcdef & 3,08 & & & \\
\hline 59 & 14,73 & $\mathrm{~g}$ & 3,13 & & & & & & \\
\hline 69 & 13,27 & abcdefg & 0,87 & & & & & & \\
\hline 78 & 14,53 & fg & 7,83 & & & & & & \\
\hline 86 & 13,73 & bcdefg & 6,06 & & & & & & \\
\hline 97 & 14,40 & defg & 2,41 & & & & & & \\
\hline 114 & 14,33 & cdefg & 2,90 & & & & & & \\
\hline
\end{tabular}

Medias seguidas de letras distintas indican diferencias, según prueba de Duncan $(P<0,05)$. T1 $=$ fruto almacenado a $3^{\circ} \mathrm{C}$ y humedad relativa del $85 \%$; $22=$ fruto almacenado a $11^{\circ} \mathrm{C}$ y humedad relativa del $80 \%$; T3 = fruto almacenado a $18^{\circ} \mathrm{C}$.

TABLA 5 - Valores medios de la relación SST/AT de la pera variedad Triunfo de Viena, bajo tres condiciones de almacenamiento.

\begin{tabular}{|c|c|c|c|c|c|c|c|c|c|}
\hline \multirow{3}{*}{$\frac{\text { DíA }}{1}$} & \multicolumn{3}{|c|}{ T1 } & \multicolumn{3}{|c|}{ T2 } & \multicolumn{3}{|c|}{ T3 } \\
\hline & \multicolumn{2}{|c|}{ Media } & \multirow{2}{*}{$\begin{array}{c}\mathbf{C V}, \mathbf{\%} \\
9,88\end{array}$} & \multicolumn{2}{|c|}{ Media } & \multirow{2}{*}{$\frac{\mathbf{C V}, \mathbf{\%}}{9,88}$} & \multicolumn{2}{|c|}{ Media } & \multirow{2}{*}{$\frac{\mathbf{C V}, \mathbf{\%}}{9,88}$} \\
\hline & 40,89 & $\mathrm{a}$ & & 40,89 & $\mathrm{a}$ & & 40,89 & $\mathrm{a}$ & \\
\hline 12 & 41,39 & $\mathrm{a}$ & 1,70 & 56,21 & bcdefg & 12,85 & 53,85 & abcdef & 16,69 \\
\hline 19 & 47,33 & $a b c$ & 4,01 & 52,63 & abcde & 7,56 & 71,24 & $\mathrm{~h}$ & 6,93 \\
\hline 26 & 41,03 & $\mathrm{a}$ & 8,76 & 59,57 & cdefgh & 8,30 & 63,12 & defgh & 8,31 \\
\hline 33 & 44,39 & $a b$ & 10,02 & 60,00 & cdefgh & 12,40 & 65,73 & fgh & 12,93 \\
\hline 41 & 41,21 & $\mathrm{a}$ & 10,14 & 60,75 & defgh & 15,61 & & & \\
\hline 50 & 52,38 & abcde & 6,27 & 68,71 & $\mathrm{gh}$ & 6,97 & & & \\
\hline 59 & 50,15 & abcd & 13,62 & & & & & & \\
\hline 69 & 59,58 & cdefgh & 15,93 & & & & & & \\
\hline 78 & 64,11 & efgh & 3,31 & & & & & & \\
\hline 86 & 63,70 & efgh & 19,84 & & & & & & \\
\hline 97 & 67,30 & $\mathrm{gh}$ & 13,73 & & & & & & \\
\hline 114 & 70,42 & $\mathrm{~h}$ & 14,68 & & & & & & \\
\hline
\end{tabular}

Medias seguidas de letras distintas indican diferencias, según prueba de Duncan $(P<0,05)$. T1 $=$ fruto almacenado a $3^{\circ} \mathrm{C}$ y humedad relativa del $85 \% ; \mathrm{T} 2=$ fruto almacenado a $11^{\circ} \mathrm{C}$ y humedad relativa del $80 \%$; $\mathrm{T} 3=$ fruto almacenado a $18^{\circ} \mathrm{C}$.

Para la condición de $3^{\circ} \mathrm{C}$ y $85 \%$ de humedad relativa, se presentan variaciones menores hasta el día 59, para luego presentar diferencias significativas, respecto al tiempo de almacenamiento. Situaciones similares fueron reportadas por Calvo (2004) y Coutinho et al. (2003) para las variedades Williams y Carrick, respectivamente.

Para las diferentes condiciones de almacenamiento, se observa un aumento en el contenido de SST a medida que el fruto alcanza su madurez organoléptica, y después decrece con la senescencia (Tabla 4). Este parámetro no muestra una gran dispersión a lo largo del almacenamiento y los valores encontrados concuerdan con los reportados por otros autores (Coutinho et al., 2003; Lombardi et al., 2000). Por otra parte, los mayores valores encontrados para la AT y los SST se presentaron en momentos cercanos al climaterio. En términos generales, la AT disminuye con el tiempo de almacenamiento mientras los SST aumentan, situación normal, ya que el producto pierde turgencia debido a la transformación de las propectinas y a la pérdida de agua, reflejada en la continua disminución del peso del producto (Pantastico, 1979).

La relación SST/AT es creciente para todas las condiciones de almacenamiento, como se observa en la Tabla 5, sin verificarse 
diferencias significativas para los primeros días en las diferentes condiciones de almacenamiento, pero haciéndose evidentes en días posteriores. De igual manera que la IR, la relación SST/AT es baja y relativamente constante cuando el producto se almacena a bajas temperaturas, presentando poca dispersión, debido a que con bajas temperaturas se presenta una mayor y completa hidrólisis del almidón (Seibert et al., 2000). Los mayores valores de la relación SST/AT se presentan a mayor tiempo de almacenamiento, baja humedad relativa y altas temperaturas.

\section{CONCLUSIONES}

1. La pera variedad Triunfo de Viena es un fruto climatérico, cuyo punto de máxima intensidad respiratoria puede desplazarse en el tiempo en la medida que la temperatura de almacenamiento disminuye.

2. La temperatura es un factor que afecta la durabilidad de la pera variedad Triunfo de Viena, presentando una relación directa con la intensad respiratoria y una relación inversa con la firmeza de la pulpa y la pérdida de peso, donde el almacenamiento a $3^{\circ} \mathrm{C}$ y humedad relativa del $85 \%$, es una alternativa para la conservación de este producto, siendo necesario realizar estudios para establecer el tiempo adecuado con el fin de evitar daños por frío, principalmente el oscurecimiento de la pulpa.

3. La relación SST/AT fue un parámetro diferencial ya que obtuvo una mayor relación de madurez para las peras almacenadas a mayor temperatura, que para las almacenadas a la temperatura más baja, lo cual lleva a concluir que las peras almacenadas a temperatura ambiente maduran más rápido y por lo tanto llegan más rápidamente a la senescencia.

4. La intensidad respiratoria, la pérdida de peso, la relación SST/AT y la firmeza de la pulpa, son los parámetros que representan mejor la evolución de la madurez de las peras variedad Triunfo de Viena.

\section{REFERENCIAS}

AMARANTE, C.; BANKS, N.H. Postharvest physiology and quality of coated fruits and vegetables. Horticultural Reviews, New York, v.26, p.161-238, 2001.

AMARANTE, C. BANKS, N.H.; GANESH, S. Effects or coating concentration, ripening stage, water status and fruit temperature on pear susceptibility to friction discolouration. Postharvest Biology and Technology, Netherlands, v.21, p.283-290, 2001.

BARRAGÁN, M.C.; SÁNCHEZ, L.J. Determinación de las propiedades físicas y fisiológicas de la pera variedad Triunfo de Viena, durante el periodo de precosecha, cosecha y postcosecha. 1998. $121 \mathrm{f}$. Tesis [Grado] - Facultad de Ingeniería, Universidad Nacional de Colombia, Bogotá, 1998.

BRACKMANN, A.; SERRETA, M.; HUNSCHE, M.; FRANJEN, A. Escurecimento interno de pêra asiática "Ya Li" durante o armazenamento refrigerado. Revista Brasileira de Fruticultura, Jaboticabal, v.24, n.1, p.251-253, 2002
CALVO, G. Efecto del 1-metilciclopropeno (1-MCP) en pera variedad Williams cosechadas con dos estados de madurez. Revista de Investigaciones Agropecuarias, Buenos Aires, v.33, n.2, p.3-26, 2004.

COUTINHO, E.F.; MALGARIM, M.B.; SOUZA, EDSON LUIZ de; TREPTOE, R.O. Qualidade pós-colheita da pêra (Pyrus communis L.) cultivar carrick, submetida a diferentes condições de armazenamento. Revista Brasileira de Fruticultura, Jaboticabal, v.25, n.3, p.417-420, 2003.

INSTITUTO COLOMBIANO DE NORMAS TÉCNICAS - ICONTEC. Norma GTC 66, Frutas y verduras: principios y técnicas del método de almacenamiento en atmósfera controlada. Bogotá, Colombia, 1999.

LOMBARDI, S.R.B.; MORAES, D.M. de; CAMELATTO, D. Avaliação do crescimento e da maturação pós-colheita de pêras da cultiva Shinsseiki. Pesquisa Agropecuária Brasileira, Brasília, v.35, n.12, p.2399-2405, 2000.

MA, S.S.; CHEN, P.M. Storage disorder and ripening behavior of "Doyenne du Comice" pears in relation to storage conditions. Postharvest Biology and Technology, Netherlands, v.28, p.281-294, 2003.

MWANAKI, M.C.; MATHOOKO, F.M.; MATSUZAKI, M.; HIWASA, K.; TATEISHI, A.; USHIJIMA, K.; NAKANO, R.; INABA, A.; KUBO, Y. Expression characteristics of seven members or the $\beta$ galactosidase gene family in "La France" pear (Pyrus communis L.) fruit during growth and their regulation by 1-methycyclopropene during postharvest ripening. Postharvest Biology and Technology, Netherlands, v.36, p.253-263, 2005.

PARRA C., A. Diseño de una metodología para la determinación de las características físicas y fisiológicas necesarias para el adecuado manejo cosecha y poscosecha de pera en Nuevo Colón (Boyacá). 1997. 128 f. Tesis [Grado] - Maestría en Gestión Ambiental para el Desarrollo. Pontificia Universidad Javeriana, Bogotá, 1997.

PANTASTICO, E.B. Fisiología de la postrecolección, manejo y utilización de frutas y hortalizas tropicales y subtropicales. México: Editorial CECSA, 1979.

PLANELLA V., I. Tecnología del manejo de postcosecha de frutas y hortalizas. Bogotá: IICA, 1987. (Serie Publicaciones Misceláneas de Colombia).

SCHOUTEN, R.E.; VELTMAN, R.H.; DE WILD, H.P.J.; KOOPEN, T.J.; STAAL, M.G.; TIJSKENS, L.M.M. Determination of $\mathrm{O}_{2}$ y $\mathrm{CO}_{2}$ permeance, internal respiration and fermentation for a batch of pears (cv. Conference). Postharvest Biology and Technology, Netherlands, v.32, p.289-298, 2004

SEIBERT, E.; BARRADAS, C.I.N.; ARAÚJO, P.J, de; BENDER, R.N. Efeito de Ethehon e da frigoconservação na maturação de pêras cv. Packham's Triumph. Pesquisa Agropecuária Brasileira, Brasília, v.35, n. 1, p.55-62, 2000 .

TRINCHERO, G.D.; SOIS, G.O.; COVATTA, F.; FRASCHINA, A.A. Inhibition of ethylene action by 1-methycyclopropene extends postharvest life or "Bartlett" pears. Postharvest Biology and Technology, Netherlands, v.32, p.193-204, 2004. 